\title{
Discours direct et stratégie rhétorique dans les communiqués de presse de Greenpeace International
}

Direct speech and rhetorical strategy in press releases issued by Greenpeace International

\section{Melwenn Cloarec}

\section{OpenEdition \\ Journals}

Édition électronique

URL : http://journals.openedition.org/asp/4695

DOI : $10.4000 /$ asp.4695

ISSN : 2108-6354

\section{Éditeur}

Groupe d'étude et de recherche en anglais de spécialité

\section{Édition imprimée}

Date de publication : 23 octobre 2015

Pagination : 45-65

ISSN : 1246-8185

Référence électronique

Melwenn Cloarec, «Discours direct et stratégie rhétorique dans les communiqués de presse de Greenpeace International », ASp [En ligne], 68 | 2015, mis en ligne le 01 novembre 2016, consulté le 03 novembre 2020. URL : http://journals.openedition.org/asp/4695 ; DOI : https://doi.org/10.4000/asp. 4695

Ce document a été généré automatiquement le 3 novembre 2020

Tous droits réservés 


\title{
Discours direct et stratégie rhétorique dans les communiqués de presse de Greenpeace International
}

\author{
Direct speech and rhetorical strategy in press releases issued by Greenpeace \\ International
}

Melwenn Cloarec

\section{Introduction}

Dans un article consacré au communiqué de presse qui, selon le TLFi ${ }^{1}$, constitue « une information de source généralement officielle, plus rarement non officielle, diffusée par la presse parlée ou écrite ", Yvonne McLaren et Calin Gurau (2005: 10) décrivent ainsi cet objet discursif: "Press releases are one type of business or corporate genre amongst many others ${ }^{2} »$. Cette définition, qui inscrit le communiqué de presse dans le domaine de la communication d'entreprise, est significative de l'approche généralement adoptée en analyse du discours ${ }^{3}$, où le communiqué de presse d'entreprise est décrit comme un "genre » particulier, c'est-à-dire un ensemble d'événements communicationnels partageant une structure et une visée communicative semblables, caractérisés par une certaine corrélation entre forme et sens, et produits par une communauté spécifique. John M. Swales (1990:58) définit ainsi le genre comme :

a class of communicative events, the members of which share some set of communicative purposes. These purposes are recognized by the expert members of the parent discourse community and thereby constitute the rationale for the genre. This rationale shapes the schematic structure of the discourse and influences and constrains choice of content and style. 
2 Selon les travaux en analyse du discours, les communiqués de presse d'entreprise partagent une visée commune: ils s'adressent typiquement au grand public par l'intermédiaire des journalistes (Jacobs 1998: 219; McLaren \& Gurau 2005: 12) et constituent, pour l'institution qui les produit, un moyen de maitriser l'information qui circule à son sujet (Sleurs et al. 2005 : 1253 ; Catenaccio 2008 : 9). Les communiqués de presse se caractérisent en outre par une certaine hybridité discursive, car ils cherchent à présenter l'institution en question sous son meilleur jour, tout en tenant compte de l'esprit critique de leurs lecteurs et notamment des journalistes dont l'objectif n'est pas, du moins peut-on le supposer, de relayer les éventuels efforts promotionnels d'une grande organisation. Les auteurs des communiqués de presse doivent donc habilement équilibrer information et promotion, ou «habiller» d'une certaine neutralité la dimension promotionnelle d'un communiqué de presse. Plusieurs auteurs ont suggéré que cette contrainte se reflète dans la forme typique du communiqué de presse, qui mêle passages informatifs et évaluatifs (McLaren \& Gurau 2005: 16; Catenaccio 2008: 24). Selon Paola Catenaccio (ibid. : 15), le communiqué de presse serait aussi marqué par une certaine interdiscursivité et s'inspirerait notamment de l'article de presse: en imitant le registre et la structure informationnelle de ce dernier, il vise à encourager la reprise par les journalistes de certains éléments d'information directement intégrables dans un article en cours de rédaction, sur le mode de la paraphrase.

3 Cependant, le communiqué de presse d'entreprise, qui a déjà fait l'objet de multiples travaux en analyse du discours, n'est plus l'unique représentant du genre: les institutions politiques, les organismes scientifiques et techniques, les associations à but non lucratif, ou encore les organisations non gouvernementales (ONG) en publient très régulièrement. Dans la présente étude, nous nous proposons de contribuer à une caractérisation des communiqués de presse publiés par Greenpeace, c'est-à-dire une variété spécialisée du communiqué de presse institutionnel envisagé en tant que genre discursif. Nous prenons en effet pour postulat l'idée que l'univers des ONG militantes ${ }^{4}$ constitue un domaine spécialisé, au sens où l'entend Michel Petit (2010 : \$20), à savoir :

un secteur de la société constitué autour et en vue de l'exercice d'une activité principale qui, par sa nature, sa finalité et ses modalités particulières et par les compétences particulières qu'elle met en jeu chez ses acteurs, définit la place reconnaissable de ce secteur au sein de la société et d'un ensemble de ses autres secteurs et détermine sa composition et son organisation spécifiques.

4 Si l'on applique cette définition à Greenpeace, il est patent qu'une ONG militante s'organise autour d'une activité centrale : peser sur les décisions des acteurs privés et publics en jouant de son influence, en déclenchant des campagnes médiatiques, ou en menant des actions directes de nature spectaculaire. Ces activités confèrent à une ONG militante telle que Greenpeace et à ses membres un statut à part dans la société, et déterminent son fonctionnement et son organisation (partage des tâches, découpage en départements, présence d'un organigramme précis...). Il est donc loisible de considérer les communiqués de presse produits par cette organisation comme un genre discursif spécialisé.

5 Au sein du paysage des ONG militantes, Greenpeace nous a semblé constituer un bon objet d'étude, car l'organisation mène une "activité intense sur le front de l'information " (Derville 1997 : 593) et produit notamment beaucoup de communiqués de presse depuis son bureau de Londres. Notre choix a également été influencé par la renommée de Greenpeace et par sa grande efficacité en matière de communication. Il 
est possible que son succès ait encouragé d'autres organisations à la prendre pour modèle, et notamment à s'inspirer de sa stratégie de communication. Greenpeace contribuerait alors à modeler le discours militant d'autres acteurs et constituerait ainsi un objet d'étude stratégique en la matière. Ce n'est bien sûr qu'une hypothèse, qui demanderait, pour être vérifiée, une étude comparative entre plusieurs organisations.

Notre première lecture des communiqués de presse de Greenpeace nous a amenée à constater l'omniprésence des citations ${ }^{5}$ au discours direct. Le discours direct est une forme de discours rapporté qui, comme l'indique Caroline Peynaud (2011:4), "se caractérise par la présence de guillemets pour la phrase entière et d'un verbe introducteur ». Du fait de leur part écrasante dans le corpus, les citations au discours direct nous ont semblé constituer un bon angle d'analyse pour tenter d'obtenir un premier aperçu sur les traits saillants du corpus, et notamment sur sa visée communicationnelle.

7 Nous présentons en premier lieu le corpus sélectionné ainsi que la méthode retenue pour son analyse. Dans notre deuxième partie, nous montrons que les citations au discours direct peuvent être considérées comme un trait caractéristique des communiqués de presse de l'organisation et qu'elles présentent une certaine hybridité discursive : bien qu'elles soient attribuées à des personnalités distinctes de l'auteur du communiqué, celui-ci intervient dans leur retranscription, voire dans leur création. Notre troisième partie est consacrée à la première fonction manifeste des citations au discours direct dans notre corpus : favoriser la reprise et la diffusion du communiqué par les journalistes en asseyant la crédibilité du discours et en imitant la structure informationnelle des articles de presse. Nous montrons en quatrième partie que les citations au discours direct servent également une visée persuasive et jouent notamment sur le pathos pour rallier le lecteur aux causes de l'organisation.

\section{Description du corpus sélectionné et de la méthode adoptée}

\subsection{Le corpus sélectionné}

8 Notre corpus de communiqués de presse recense 200 items publiés entre le 27/10/2011 et le 24/10/2013 sur le site international de Greenpeace (Greenpeace International'); ils sont disponibles dans la rubrique "Press Centre ». Après suppression des annexes, des adresses URL et des éléments n'appartenant pas au corps du communiqué (les mentions "On this page » et "Press release ", l'annonce de la date et du lieu d'écriture et la section "Tags and Categories » qui énumère les mots-clés du communiqué), notre corpus de travail totalise 95698 mots. Les 200 items rassemblés dans notre corpus traitent de chacune des grandes sphères d'action de l'organisation définies sur le site de Greenpeace International ${ }^{7}$ : le combat contre la surpêche et le réchauffement climatique et, pour l'agriculture durable, la protection des forêts, le désarmement nucléaire et la suppression de produits toxiques. Afin de faciliter les références aux communiqués de presse dans notre article, nous avons numéroté les items de notre corpus : le communiqué le plus récent porte le numéro 1 tandis que le communiqué le plus ancien porte le numéro 200. Cette numérotation correspond à l'ordre dans lequel apparaissent les communiqués de presse sur le site de Greenpeace International. 
9 Nous avons rassemblé les 564 citations au discours direct, qui totalisent 27728 mots et représentent $29 \%$ du corpus, dans un même sous-corpus. Nous avons également constitué un sous-corpus de citations au discours indirect rassemblant 107 citations et 2423 mots, à des fins de comparaison.

\subsection{Le cadre d'analyse}

10 Notre étude des citations au discours direct dans les communiqués de presse s'inscrit dans le cadre de l'analyse argumentative. Dans le Traité de l'Argumentation, Chaïm Perelman et Lucie Olbrechts-Tyteca (1970:12) indiquent: «l'objet de [la théorie de l'argumentation] est l'étude des techniques discursives permettant de provoquer ou d'accroître l'adhésion des esprits aux thèses qu'on présente à leur assentiment ». Raphaël Micheli (2007: 68) précise que l'analyse argumentative aborde le discours argumentatif d'un point de vue non normatif: on ne cherche pas à "départager les argumentations rationnelles des argumentations fallacieuses »; à l'inverse, il s'agit de saisir la dimension proprement langagière du phénomène argumentatif. L'analyse argumentative s'inspire abondamment de la tradition rhétorique et utilise certains de ses concepts opératoires «dans l'optique d'une description des pratiques discursives modernes".

\section{Les citations dans les communiqués de presse : un statut énonciatif ambigu}

\subsection{Citations authentiques et «pseudo-citations»}

11 On distingue souvent, en analyse du discours, les citations au discours direct du discours produit par l'énonciateur. Selon Patrick Charaudeau (2005: 134), les propos rapportés au discours direct sont émis par un locuteur primaire, distinct du locuteur secondaire qui mentionne la citation: "le discours est reproduit tel quel, avec une certaine autonomie. Le locuteur primaire est souvent précisé ». De même, C. Peynaud (2011: 2), dans un article portant sur le rôle des citations dans le discours journalistique, qualifie «le discours produit par le journaliste» de "discours encadrant » et l'oppose aux citations, qui constituent le " discours encadré ».

12 Il en va traditionnellement autrement dans les communiqués de presse, qui, selon Geert Jacobs (1994: 237), ne rapportent bien souvent que des "pseudo-citations ", rédigées ad hoc par les auteurs des communiqués de presse : «Generally, the quoted words are almost never certainly verbalized by the named source, but written by a press officer and (possibly) approved by the source [...]».

\subsection{Le discours direct dans notre corpus : citations et « pseudo- citations "}

Les citations au discours direct qui apparaissent dans notre corpus sont-elles authentiques ou peuvent-elles être considérées comme des «pseudo-citations »? Il est souvent difficile d'établir cette distinction, mais plusieurs indices plaident en faveur d'une forte densité de «pseudo-citations ». 

paraissent certes authentiques. Les propos des personnalités externes à l'organisation (hommes et femmes politiques, scientifiques...) sont fidèlement traduits dans les communiqués, puisqu'il est illégal de prêter de faux propos à autrui, au risque de s'exposer à des poursuites pour diffamation. Le communiqué 7 reproduit ainsi de façon apparemment fidèle les propos de V. Poutine au sujet de l'équipage de l'Arctic Sunrise ${ }^{8}$ («It is absolutely evident that they are of course, not pirates ») : les sites de la BBC, d'Al Jazeera et de Reuters 9 reprennent la phrase à l'identique.

15 Les auteurs des communiqués insistent parfois sur l'authenticité du discours retranscrit lorsqu'ils citent des militants susceptibles d'émouvoir le lecteur par leur propos (nous développons cette idée en quatrième partie). Ainsi, les communiqués de presse reprennent parfois les formes réduites des verbes, caractéristiques d'un discours oral: «That's why we're here, to let the public and Formula One fans know what this company is really up to " (CP39).

Cependant, une proportion importante des citations dans notre corpus semble dotée d'un statut énonciatif ambigu. Les auteurs des communiqués restent tout d'abord très évasifs quant à la source de la citation, comme ici : "In a statement, Greenpeace International said» (CP11); de plus, ils passent souvent sous silence les circonstances dans lesquelles les propos rapportés ont été prononcés, comme dans l'exemple suivant : «Vladimir Chuprov of Greenpeace Russia said...» (CP2 - aucune précision n'est apportée dans ce texte sur les circonstances de cette prise de parole). Ces imprécisions sèment le doute sur l'authenticité des citations.

17 Autre indice troublant: on trouve de nombreux échos entre des citations pourtant attribuées à des personnes différentes. Les propos officiellement formulés par Ben Ayliffe dans le communiqué de presse 32 ressemblent ainsi fortement au discours prononcé par Faiza Oulahsen dans le communiqué de presse 33 :

Let's be absolutely clear about this: the real threat to the Arctic comes not from Greenpeace International but from oil companies like Gazprom that are determined to ignore both science and good sense to drill in remote, frozen areas (CP32)

Let's be absolutely clear: Arctic drilling is not safe and oil spills are inevitable. The real risk to the Arctic is not from peaceful protest but from reckless oil companies like Shell and Gazprom (CP33)

Enfin, l'auteur du communiqué de presse se cite parfois lui-même : c'est par exemple le cas dans les communiqués 36 et 37 , où les propos rapportés sont attribués à Christy Ferguson, qui signe les communiqués de presse depuis le passage du Nord-Est.

Hormis les citations attribuées à des personnalités extérieures à l'organisation et les deux exemples que nous avons mentionnés plus tôt, nous formons l'hypothèse, sans toutefois être en mesure de le prouver de manière définitive, que les citations qui apparaissent dans notre corpus semblent pouvoir être attribuées aux auteurs de nos communiqués plutôt qu'aux sources désignées et que leur statut énonciatif ambigu peut éclairer les visées communicationnelles des communiqués de presse de Greenpeace et les moyens mis en œuvre pour les atteindre. 


\section{L'emploi du discours direct favorise la reprise et la diffusion du communiqué par les journalistes}

\subsection{Discours direct, crédibilité et diffusion du discours militant}

20 d'abord à donner aux communiqués une apparence objective, pour renforcer leur crédibilité et favoriser leur reprise par les journalistes dans leurs propres articles. Dire que le discours direct contribue à l'apparente neutralité du communiqué semble à première vue contradictoire, étant donné que les personnes citées incarnent une idée ou un positionnement, ce qui tend normalement à personnaliser l'argumentaire. De fait, les pronoms we (298 occurrences ; $0,3 \%$ du corpus) et $I$ ( 77 occurrences ; $0,08 \%$ du corpus), à une exception près, ne sont utilisés que dans les citations et non dans le corps du communiqué de presse, où l'on parle pour l'essentiel de l'organisation à la troisième personne, comme dans le communiqué de presse 1: "Greenpeace International is calling for them to be immediately released». Les modaux et semimodaux ${ }^{10}$ traduisent également le point de vue de l'énonciateur: comme l'indique Janine Bouscaren (1991: 11), «la modalité marque la position de l'énonciateur par rapport à la validation de la relation prédicative [ou] par rapport au co-énonciateur ou à d'autres sujets ». Or, comme le montre l'annexe 1 , les modaux et semi-modaux sont nettement plus fréquents dans les citations (ils représentent 1,7\% du corpus de citations) que dans le reste des communiqués (ils représentent $0,7 \%$ du corpus de communiqués sans citations).

21

Les citations, par ailleurs, s'éloignent du ton neutre et de la dimension factuelle des communiqués et sont le lieu où s'expriment des propos évaluatifs et engagés : les membres de l'organisation cités dans les communiqués adoptent en effet un ton catégorique, qui se manifeste dans notre corpus par un style hyperbolique jouant sur plusieurs procédés. Les citations multiplient ainsi les recours aux adverbes d'intensité : comme le montre l'annexe 2 , un peu moins de la moitié des adverbes d'intensité comptabilisés dans notre corpus $(48,3 \%)$ apparaissent dans les citations, qui représentent moins du tiers (29\%) du corpus. Dans les exemples suivants, Vladimir Chuprov, membre de Greenpeace Russia, et Kumi Naidoo, directeur exécutif de Greenpeace International, utilisent ces procédés pour dénoncer la faible capacité d'analyse des adversaires de l'organisation: «[Hooliganism] is a serious and wildly disproportionate charge » (CP2); « There is absolutely no justification for any charge of piracy, or the decision to deny bail » (CP20). L'emploi du déterminant no est également significatif puisque, à l'inverse de la négation plus fréquente not... any, le déterminant permet de «donner plus de force à ce qu'on dit » (Larreya \& Rivière 2005 : 188). Cette opération de négation est préférée à not... any dans l'ensemble du corpus (on compte 119 négations formées à l'aide de no contre six négations construites avec not... any) ${ }^{11}$, mais avant tout dans les citations, qui concentrent $42,9 \%$ des négations en no tout en représentant moins du tiers du corpus; elles ne comportent par ailleurs aucune négation en not... any. On lit notamment dans le communiqué de presse 19: «Any charge of piracy against peaceful activists who tried to protect the Arctic environment has no merit in international law $»$.

Nous formons malgré tout l'hypothèse que ces citations évaluatives et partisanes contribuent à donner une apparence de neutralité aux communiqués de presse. Nous 
nous rallions ici à l'idée formulée par Colleen Cotter (2010: 146), pour qui «le fait de citer quelqu'un ou d'utiliser les mots d'autrui permet un positionnement distant ou 'distal' du journaliste vis-à-vis du contenu et des sources d'information $»^{12}$. La citation est un procédé habile qui permet à l'auteur du communiqué de véhiculer des idées et des points de vue sans les prendre en charge. Par ailleurs, le caractère engagé des citations souligne, par contraste, l'absence de parti pris dans le corps des communiqués.

Les «amorces » utilisées par les auteurs des communiqués pour rapporter ces propos témoignent également de leur volonté de préserver la neutralité apparente des communiqués. Comme le montre le tableau 1 ci-dessous, 349 des 564 citations au discours direct que compte notre corpus sont rapportées au moyen du verbe introducteur said; le concurrent de said comptant le plus d'occurrences après says (à connotation neutre lui aussi) est according to qui n'apparaît qu'à quatre reprises avec des citations (added, continued et concluded ne peuvent pas être considérés comme des concurrents de said, puisqu'ils n'apparaissent qu'après une première citation introduite par said).

Tableau 1. Fréquences des verbes ou expressions introduisant les citations au discours direct dans les communiqués de presse de Greenpeace

\begin{tabular}{|l|l|}
\hline Verbe introducteur ou expression introductrice & $\begin{array}{l}\text { Occurrences en accompagnement de } \\
\text { citations }\end{array}$ \\
\hline said & 349 \\
\hline added & 29 \\
\hline continued & 10 \\
\hline says & 9 \\
\hline concluded & 6 \\
\hline according to & 4 \\
\hline made the following statement & 4 \\
\hline saying & 3 \\
\hline warned & 2 \\
\hline responded & 1 \\
\hline admits & 1 \\
\hline asked & 1 \\
\hline commented & 1 \\
\hline delivered the following statement & 1 \\
\hline
\end{tabular}




\begin{tabular}{|l|l|}
\hline stated & 1 \\
\hline tells & 1 \\
\hline warns & 1 \\
\hline warned & 1 \\
\hline writes & 1 \\
\hline wrote & 1 \\
\hline $\begin{array}{l}\text { Pas de verbe introducteur ou d'expression } \\
\text { introductrice }\end{array}$ & 137 \\
\hline
\end{tabular}

Or, comme l'indique C. Cotter (ibid. : 149), said est le verbe introducteur le plus neutre : "The quoting verb, because of its purported neutrality, with very few exceptions, is 'said' [...]. Any other verbs are disfavored and suggest a deviation from the norm ».

Cet effort de neutralisation du discours participe probablement d'une stratégie de crédibilisation des communiqués de presse. En effet, ce procédé est souvent adopté par les instances engagées dans un "processus de construction de crédibilité " (Charaudeau \& Maingueneau 2002: 44). L'attitude de "neutralité», qui amène l'énonciateur «à effacer dans son discours toute trace de jugement ou d'évaluation personnelle » constitue l'un des trois types de positionnements discursifs destinés à établir la crédibilité d'une instance identifiés par P. Charaudeau (2009 : \$28).

Si les auteurs des communiqués cherchent autant à présenter leur discours comme crédible, c'est peut-être parce que ce dernier semble être principalement destiné à des lecteurs critiques, à savoir les journalistes, qui ont le pouvoir de reprendre le communiqué dans leurs publications pour lui permettre d'atteindre un public plus large. Les journalistes constituent le relais traditionnel des organisations auprès du grand public et la cible principale des communiqués de presse. Plusieurs auteurs, cependant, notent que cet intermédiaire est devenu moins nécessaire avec l'essor d'Internet : tout internaute a désormais la possibilité d'accéder aux communiqués de presse de l'organisation publiés en ligne. G. Jacobs et I. Strobbe (2005: 290) font ainsi remarquer:

serve a key-function in turning press releases into more or less public documents and in reducing the gatekeeping role traditionally played by journalists in handling paper press releases ». P. Catenaccio $(2008: 15)$ indique également :

With companies and institutions now almost invariably including press and media sections in their websites, [...] press release writers now have an opportunity to entirely bypass journalistic intervention in reaching the public.

Les communiqués de presse de Greenpeace, accessibles en ligne et par conséquent à tous, n'en paraissent pas moins destinés en priorité aux journalistes. P. Catenaccio (2007: 162) suggère tout d'abord que la mention d'un numéro de téléphone dans les communiqués de presse montre l'ouverture de l'organisation aux journalistes : elle leur offre ainsi la possibilité de la contacter pour obtenir des informations supplémentaires. Or, comme le montrent les captures d'écran en figure 1, les communiqués de presse sont systématiquement dotés d'une section « Contacts », dans laquelle apparaît non pas 
93) indique en effet: "newspaper journalists are critical users of release materials ", tandis que Lewis et al., cités par P. Catenaccio et al. (2011: 1848), notent: " the use of news agency wire-service copy is more acceptable to journalists than the use of public relations material ». Pour être repris par un journaliste, le communiqué se doit d'être (apparemment) objectif, comme l'écrit P. Catenaccio (2008: 14) : "public relation practitioners argue that the less a press release manifests itself as promotional, the more it is likely to be used by journalists, and therefore the more potentially promotional it becomes». Nous soutenons que le discours direct, qui s'inscrit paradoxalement dans l'«attitude de neutralité » adoptée dans les communiqués de presse de Greenpeace, encourage leur reprise par les journalistes et leur diffusion auprès du grand public et accroît ainsi leur efficacité.

\subsection{Discours direct, mimétisme et diffusion du discours militant}

30 La présence de citations au discours direct dans les communiqués de presse peut également favoriser leur reprise par les journalistes, non seulement parce que cela leur donne une apparence neutre, mais parce que les citations, composantes typiques des articles de presse, donnent aux communiqués de presse un tour pseudo-journalistique.

31 En analyse du discours, les citations ont souvent été décrites comme un trait typique du discours journalistique. C. Peynaud (2011:2) insiste ainsi sur "l'hétérogénéité » du discours journalistique, qui a pour caractéristique d'intégrer des "éléments extérieurs». L'auteur cite Sophie Moirand qui indique: "Le texte journalistique devient alors une mosaïque de voix, constituée d'une pluralité de fils intertextuels, et le fil horizontal du discours apparait, dans sa matérialité même, fracturé par des marques de cette hétérogénéité " (ibid.). De même, C. Cotter (2010: 147), dans son étude des conventions journalistiques, fait remarquer: "Throughout the story, the quotes are positioned to support the news angle and to support the lead "; « a sufficient number of attributed quotes or sources is expected (a minimum of two or three sources)».

L'importance des citations dans notre corpus et l'absence de commentaire sur les citations, introduites la plupart du temps au moyen du verbe said, rapprochent les items de notre corpus des articles de presse. Les citations au discours direct apparaissent de plus au même endroit dans les articles de presse et dans les communiqués de Greenpeace, ce qui accentue leur ressemblance. C. Cotter (ibid.) souligne ainsi la rareté des citations dans les chapeaux introductifs des articles de presse : "well-written stories do not generally begin with quotes". Cela se retrouve dans les communiqués de presse de Greenpeace : seuls treize chapeaux introductifs sur 200 comportent des citations, et seulement deux d'entre eux (CP184, CP197) commencent par une citation.

33 L'adoption de conventions journalistiques, parmi lesquelles figurent les citations au discours direct, constitue un choix stratégique pour accroître les chances de diffusion des communiqués dans la presse. Les journalistes doivent en effet souvent faire face à des contraintes de temps, comme le soulignent Walters et al. (1994: 346), et auraient donc tendance à s'inspirer davantage des communiqués de presse qui adoptent déjà le format de l'article de presse. K. Sleurs et al. (2003 : 193), citant A. Bell, nous rappellent en effet : " it has been shown that, in order to facilitate this reproduction process, press releases are already prefabricated in an appropriate news style and therefore require the minimum of reworking ». 
Il nous a également semblé que la présence de citations au discours direct, en plus d'accroître les chances des communiqués d'être diffusés par la presse, leur permettrait d'être mieux diffusés. Les idées de l'organisation sont soigneusement présentées dans les communiqués de presse : elles doivent donc être retranscrites aussi fidèlement que possible pour avoir l'effet escompté par la source énonciative. Nous nous rangeons ici à l'hypothèse avancée par T. Walters et al. (1994:347):

[T]he release has been written exactly to express the thoughts, needs and issues of the institution on whose behalf it was produced. Manipulation by the reporter of syntactical elements of a press release may change its meaning and, hence, its impact on and value to that institution. Thus, public relations practitioners would like to keep such changes to a minimum. To do so, the practitioner must write the release as the reporter would.

L'imitation du style journalistique par les auteurs des communiqués de presse renvoie à ce que G. Jacobs (1994) appelle la "préformulation", soit l'incorporation des conventions journalistiques dans les communiqués pour persuader les journalistes de les reprendre aussi exactement que possible et de (mieux) diffuser le message de l'organisation. G. Jacobs (ibid. : 234) résume ainsi cette idée : « People use words to get other people to use words".

L'emploi du discours direct dans les communiqués nous semble donc être destiné à donner une apparence objective aux communiqués et ainsi renforcer la crédibilité du discours, à modeler les communiqués sur les articles de presse et, à terme, à favoriser leur reprise par les journalistes et à accroître leur efficacité. Cependant, notre étude de corpus nous a amenée à constater que ce procédé peut également permettre aux auteurs des communiqués de mettre en avant les positions de l'organisation en espérant ainsi convaincre le lecteur de s'y rallier.

\section{L'effet persuasif du discours direct}

\subsection{Entre objectivité apparente et subjectivité dissimulée}

L'emploi de citations au discours direct ne donne aux communiqués de presse qu'une apparence de neutralité : l'auteur du communiqué de presse semble également s'en servir pour pouvoir exprimer une opinion sans décrédibiliser son propos. Ce que soulignait C. Peynaud (2011:9) dans son étude des articles de presse nous semble également pertinent pour notre corpus : «Les citations [...] permettent au journaliste de rendre son point de vue moins contestable et de l'exprimer de manière moins visible ».

Les auteurs de nos communiqués de presse se servent du discours direct pour véhiculer le discours de l'organisation. En effet, comme nous le montre le tableau 2, la majorité des citations au discours direct (515 sur 564, soit 91,3\%) reprend les propos des membres de l'organisation; à l'inverse, seules quarante-neuf citations au discours direct (soit 9,7\% des citations au discours direct) retranscrivent les propos des personnalités extérieures à Greenpeace. 
Tableau 2. Sources officielles des citations au discours direct et au discours indirect

\begin{tabular}{|l|l|l|l|}
\hline Source & $\begin{array}{l}\text { Nombre de citations au discours } \\
\text { direct et proportion dans le corpus } \\
\text { de citations au discours direct }\end{array}$ & $\begin{array}{l}\text { Nombre de citations au discours } \\
\text { indirect et proportion dans le } \\
\text { corpus de citations au discours } \\
\text { indirect }\end{array}$ & TOTAL \\
\hline $\begin{array}{l}\text { Membre de } \\
\text { Greenpeace }\end{array}$ & $515(91,3 \%)$ & $23(21,5 \%)$ & 538 \\
\hline $\begin{array}{l}\text { Personnalité } \\
\text { extérieure }\end{array}$ & $49(9,7 \%)$ & $84(79,5 \%)$ & 133 \\
\hline TOTAL & 564 & 107 & 671 \\
\hline
\end{tabular}

Les citations attribuées aux membres de l'organisation sont non seulement plus nombreuses, mais aussi mieux mises en valeur. Comme le montre le tableau 2, les propos des militants de Greenpeace sont majoritairement retranscrits au discours direct, à l'inverse des propos des personnalités extérieures à l'organisation, et notamment de ses adversaires, plutôt au discours indirect. C'est par exemple le cas dans le communiqué de presse 34 où les déclarations des responsables de Herakles Farms sont rapportées au discours indirect : « Herakles Farms stated it had ceased work and was obeying the suspension in a company press release on 18 May 2013, and it was lifted on 29 May ». À l'inverse, les propos d'Irène Wabiwa, membre de Greenpeace Afrique chargée de la lutte pour la protection des forêts, y sont rapportés au discours direct, comme ici :

"The company is continuing to clear and log in the concession area, and is now marking logs in a way that suggests that they are intended for commercial sale," says Irene Wabiwa, forest campaigner for Greenpeace Africa.

Ce choix n'est pas innocent: le discours direct permet de mettre en avant certains propos par rapport à d'autres positions, que le lecteur prendra probablement moins en considération. Le discours direct, en effet, est mis en valeur à un double niveau dans les communiqués de Greenpeace: les auteurs passent à la ligne pour introduire les citations, qui occupent un paragraphe bien distinct; de plus, le discours change d'énonciateur, puisqu'il est attribué à une personnalité distincte de l'auteur du communiqué.

\subsection{Discours direct et effet de pathos}

41 Le discours direct, qui prend parfois des accents pathétiques, semble enfin être destiné à rallier les lecteurs aux causes défendues dans les communiqués de presse. Dans la langue commune, on attribue désormais un sens péjoratif au terme pathos, communément employé pour désigner un «débordement émotionnel, généralement manquant de sincérité " (Charaudeau \& Maingueneau, 2002: 425). En rhétorique, cependant, le terme ne désigne pas le sentiment, mais la stratégie discursive qui peut le provoquer. Le pathos, comme le rappelle Claire Sukiennik (2008:4) « constitue l'un des trois types d'arguments [destinés à persuader l'auditoire] dans la Rhétorique d'Aristote " (les deux autres arguments étant le logos et l'ethos). Le pathos est une stratégie 
rhétorique tournée vers l'auditoire et vise à l'émouvoir: il s'agit, pour Dominique Desmarcheliers (2005: 87), de "provoquer une tension émotionnelle chez le destinataire ", de susciter des sentiments tels que la pitié.

Selon C. Sukiennik (2008:4), l'appel à la pitié de l'auditoire est une stratégie de persuasion particulièrement efficace, du fait de son «impact émotionnel »: « l'appel à la pitié a un énorme impact émotionnel sur l'auditoire, lui faisant oublier qu'il ne possède pas les données nécessaires pour juger le cas équitablement et impartialement ».

Dans le chapitre 8 du livre II de la Rhétorique, consacré à la pitié, Aristote fournit des indications précieuses sur les stratégies rhétoriques à adopter pour susciter la pitié :

[L]a pitié sera le chagrin que nous cause un malheur dont nous sommes témoins et capable de perdre ou d'affliger une personne qui ne mérite pas d'en être atteinte, lorsque nous présumons qu'il peut nous atteindre nous-mêmes, ou quelqu'un des nôtres, et cela quand ce malheur paraît être près de nous. (traduit et cité par Sukiennik, ibid. : 2)

Les trente-deux premiers communiqués de notre corpus, consacrés à l'emprisonnement de vingt-huit militants et de deux journalistes dans l'Arctique russe à partir du 18 septembre 2013, jouent beaucoup sur le pathos susceptible de se manifester dans l'emploi du discours direct. Le recours à ce procédé s'explique peut-être par la gravité de la peine qu'encouraient les militants en Russie ${ }^{13}$ et par la nécessité pressante de susciter l'empathie et de mobiliser l'opinion internationale pour forcer leur libération.

Selon la définition d'Aristote, un individu éprouvera de la pitié s'il est amené à penser que le malheur décrit « peut [1']atteindre [lui-même] ou quelqu'un des [siens]» (traduit et cité par Sukiennik, ibid.). Or les citations au discours direct comprises dans notre sous-corpus fournissent de nombreux détails sur les militants emprisonnés afin d'établir, peut-être, une certaine proximité entre le lecteur et les activistes. Dans le communiqué de presse 8, la citation attribuée à l'un des militants emprisonnés, Frank, nous informe sur le nom de sa station de métro et sur la distance qui la sépare de son domicile: «[If] I walk round the flooded $5 \mathrm{~m} \times 5 \mathrm{~m}$ concrete pen 80 times it's the equivalent of walking [from home] to Queen's Park, which is Frank's local tube rail station in London $»$.

Les communiqués donnent également à entendre la voix des militants, dont on retranscrit ostensiblement les paroles exactes, peut-être là aussi pour tenter d'inspirer un sentiment de proximité chez le lecteur. Comme nous l'avons déjà remarqué, certains communiqués de presse s'attachent en effet à retranscrire fidèlement le parler des militants. Ils conservent par exemple les erreurs de grammaire, comme dans le passage suivant : «I'm considered to be dangerous for society, but how it can be so ?» (CP9). Norman Fairclough (cité par Tuomarla 1999: 219) parle à ce sujet de " conversationalization»: il s'agit selon lui d'un procédé de persuasion fréquemment employé dans les médias "pour créer un effet de reconnaissance et une illusion de familiarité »(ibid.).

Aristote précise également que la pitié survient lorsque nous sommes « témoins » d'un malheur. Or les citations au discours direct qui apparaissent dans le sous-corpus rapportent de façon très détaillée et visuelle les conditions d'emprisonnement des militants, de telle sorte que le lecteur a l'impression d'assister aux événements décrits. Le communiqué 6 évoque ainsi les prisonniers partageant le quotidien des militants, l'état de la prison et la routine des détenus : 
Faiza writes of being held 'in a dirty cell, alone, isolated from the rest', only able to 'catch a glimpse of other Russian prisoners in the corridor'. She says: 'Once in a while a rat crawls across the floor. (...) She also says the highlight of her day is the exercise hour, when she 'walks around in a dark concrete space of about $5 \times 5$ meters, where you're lucky if you can see the sky through the cracks in the rotten and leaky roof.' (CP6)

\section{Conclusion}

Cette étude a révélé les spécificités des citations au discours direct comprises dans les communiqués de presse de Greenpeace et nous a permis d'entrevoir quelques caractéristiques saillantes du corpus.

Ces citations se distinguent des citations habituelles, c'est-à-dire des retranscriptions, dans un premier discours, d'un discours extérieur attribué à un énonciateur distinct de l'énonciateur du premier discours, de façon totale ou partielle. Les citations comprises dans notre corpus correspondent en effet aux "pseudo-citations » que l'on retrouve fréquemment dans les communiqués de presse d'entreprise : l'auteur du communiqué ne se contente pas de rapporter des propos ; il est lui-même, à des degrés variables, l'auteur de la citation.

Il y a donc une parité d'emploi entre les communiqués de presse d'entreprise et ceux de Greenpeace. Cependant, leurs visées communicationnelles contribuent à distinguer ces deux sous-genres. Les citations au discours direct comprises dans les communiqués de presse de Greenpeace semblent en effet avoir deux fonctions : elles sont tout d'abord utilisées pour encourager la reprise des communiqués par les journalistes et, ainsi, assurer leur diffusion et leur efficacité. Pourtant engagées et virulentes, les citations au discours direct contribuent paradoxalement à donner une apparence de neutralité au communiqué de presse ; elles rapprochent par ailleurs les communiqués des articles de presse, lesquels utilisent abondamment les citations au discours direct. Ces deux procédés semblent être destinés à convaincre les journalistes critiques et pressés par le temps de reprendre les communiqués aussi exactement que possible. Les citations au discours direct, qui mettent en valeur les propos des militants et jouent parfois sur le pathos, sont également censées rallier le lecteur aux causes défendues par l'organisation.

51 Les citations au discours direct sont donc significatives d'une stratégie double à l'œuvre dans les communiqués de Greenpeace: jouer sur la neutralité et sur l'emprunt de conventions journalistiques pour mieux faire adopter son discours, tout en appelant le lectorat à défendre l'environnement aux côtés des militants. Notre étude a cependant montré le caractère limité et occasionnel du recours au pathos dans les citations, peutêtre à cause de la contradiction latente entre cette stratégie et la priorité manifeste des communiqués : présenter Greenpeace comme une organisation professionnelle et digne de confiance, ce qui implique l'adoption d'un style simple et neutre.

L'entremêlement de ces deux visées communicationnelles semble démarquer les communiqués de presse de Greenpeace des communiqués de presse d'entreprise qui, tels qu'ils sont décrits dans les travaux de recherche existants, sont destinés à persuader le lecteur de la crédibilité du discours tenu, mais ne l'encouragent guère à passer à l'action aux côtés de l'entreprise. Il serait intéressant d'élargir notre analyse à l'ensemble des communiqués (au lieu de nous concentrer sur les citations seules) et aux communiqués de presse publiés par d'autres organisations militantes pour vérifier si 
leurs auteurs ont eux aussi recours au pathos. Cette étude nous aiderait à déterminer si les communiqués de presse des organisations militantes constituent un sous-genre particulier au sein du genre « communiqués de presse».

\section{BIBLIOGRAPHIE}

BOUSCAREN Janine. 1991. Linguistique anglaise : initiation à une grammaire de l'énonciation. Paris : Ophrys.

CATENACCIO, Paola. 2007. «Because crisis happens: Analysing the implicit in Parmalat's crisis press release ». ILCEA 9, 153-170.

CATENACCIO, Paola. 2008. « Press releases as hybrid genre: Addressing the informative/ promotional conundrum ». Pragmatics 18, 9-31.

CATENACCIO, Paola et alii. 2011. « Towards a linguistics of news production ». Journal of Pragmatics $43,1843-1852$.

ChARAUdeAU, Patrick. 2005. Le Discours politique : les masques du pouvoir. Paris : Vuibert.

CHARAUDEAU, Patrick. 2009. «Identité sociale et identité discursive. Un jeu de miroir fondateur de l'activité langagière », consulté le 23 avril 2014 <http://www.patrick-charaudeau.com/Identitesociale-et-identite.html>.

Charaudeau, Patrick \& Dominique Maingueneau (dir.). 2002. Dictionnaire d'analyse du discours.

Paris : Seuil.

COTTER, Colleen. 2010. News Talk: Investigating the Language of Journalism. Cambridge : Cambridge University Press.

DALE, Stephen. 1996. McLuhan's children: the Greenpeace message and the media. Toronto : Between the Lines.

DERVILLE, Gregory. 1997. « Le combat singulier Greenpeace-SIRPA. La compétition pour l'accès aux médias lors de la reprise des essais nucléaires français ». Revue française de science politique 5, 589629.

DESMARCHELIERS, Dominique. 2005. « De la propagande à la communication politique ». In Banks, D. (dir.), Aspects linguistiques du texte de propagande. Paris : L'Harmattan, 83-97.

JACOBS, Geert. 1994. «Formulations and preformulations in press releases ». In GALUYKENS, A. \& K. PelSMAeKers (dir.), Discourse in Professional Contexts. Munich : Lincom Europa, 229-245.

JACOBS, Geert. 1998. « Self-reference in press releases ». Journal of Pragmatics 31, 219-242.

JACOBS, Geert \& Kim SLEURS. 2005. «Beyond preformulation: an ethnographic perspective on press releases ». Journal of Pragmatics 37, 1251-1273.

JACOBS, Geert \& Ilse STROBBE. 2005. «E-releases, a view from linguistic pragmatics ». Public Relations Review 31, 289-291. 


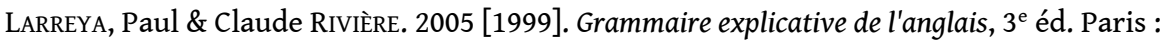
CampusPress.

LEECH, Geoffrey. 2004. « Recent grammatical change in English: data, description, theory ». In AIJMER, K. \& B. ALTENBERG (dir.), Language and Computers, Advances in Corpus Linguistics. Papers from the 23rd International Conference on English Language Research on Computerized Corpora (ICAME 23), 61-81.

MCLAREN, Yvonne \& Calin GURAU. 2005. « Characterising the genre of the corporate press release ». LSP and Professional Communication 5/1, 10-19.

MicheLI, Raphaël. 2007. « Stratégies de crédibilisation de soi dans le discours parlementaire ». A Contrario 5, 67-84.

PANDER MAAT, Henk. 2008. «Editing and genre conflicts: how newspaper journalists clarify and neutralize press release copy ». Pragmatics 18, 87-113.

PERELMAN, Chaïm \& Lucie OLBRECHTS-TYTECA. 1970. Traité de l'Argumentation. Bruxelles : Éditions de l'Institut de Sociologie.

PeTiT, Michel. 2010. « Le discours spécialisé et le spécialisé du discours ». E-rea, Revue électronique d'études sur le monde anglophone 8/1, consulté le 17 décembre $2013<\mathrm{http}$ :// erea.revues.org/1400>.

Peynaud, Caroline. 2011. «Parole rapportée et positionnement discursif dans la presse américaine : analyse de l'utilisation des citations dans des commentaires politiques ». ASp 59, 4364, consulté le 15 octobre 2014 <http://asp.revues.org/3047>.

SLEURS, Kim, Geert JACOBS \& Luuk VAN WAES. 2003. « Constructing press releases, constructing quotations: a case study ». Journal of Sociolinguistics 7/2, 192-212.

SUKIENNIK, Claire. 2008. « Pratiques discursives et enjeux du pathos dans la présentation de l'Intifada al-Aqsa par la presse écrite en France ». Argumentation et Analyse du discours 1, consulté le 13 octobre 2014 <http://aad.revues.org/338>.

TuOMARLA, Ulla. 1999. « Le discours direct de la presse écrite : un lieu de l'oralisation de l'écrit ». Faits de langue 7/13, 219-229.

SWALES, John M. 1990. Genre Analysis. Cambridge : Cambridge University Press.

WALTERS, Timothy N., Lynne M. WALTERS \& Douglas P. STARR. 1994. « After the highwayman: syntax and successful placement of press releases in newspapers ». Public Relations Review 20/4, 345-356.

\section{ANNEXES}

Annexe 1 : Modaux et semi-modaux dans le corpus des communiqués sans citations et dans le corpus des citations

\begin{tabular}{|l|l|l|l|l|l|}
\hline & \multirow{2}{*}{} & \multicolumn{3}{|l|}{ Corpus de communiqués sans citations } & \multicolumn{2}{l|}{ Corpus de citations } \\
\cline { 3 - 6 } & & Nombre & Proportion & Nombre & Proportion \\
\hline \multirow{2}{*}{ Modaux } & CAN & 157 & $0,2 \%$ & 89 & $0,3 \%$ \\
\cline { 3 - 6 } & & &
\end{tabular}




\begin{tabular}{|c|c|c|c|c|c|}
\hline & COULD & 47 & $0,05 \%$ & 18 & $0,06 \%$ \\
\hline & MAY & 18 & $0,02 \%$ & 5 & $0,02 \%$ \\
\hline & MIGHT & 2 & do,002\% & 5 & $0,02 \%$ \\
\hline & MUST & 60 & $0,06 \%$ & 98 & $0,3 \%$ \\
\hline & WILL & 252 & $0,3 \%$ & 146 & $0,5 \%$ \\
\hline & WOULD & 109 & $0,1 \%$ & 29 & $0,1 \%$ \\
\hline & SHALL & 2 & D0,002 \% & 0 & $\mathrm{X}$ \\
\hline & SHOULD & 21 & $0,02 \%$ & 61 & $0,2 \%$ \\
\hline \multirow{9}{*}{ Semi-modaux } & OUGHT TO & $\mathrm{X}$ & $\mathrm{X}$ & 1 & do,004\% \\
\hline & HAVE TO & 11 & $0,01 \%$ & 10 & $0,04 \%$ \\
\hline & BE TO & 5 & D0,005 \% & 2 & d0,007\% \\
\hline & BE GOING TO & 1 & D0,001 \% & 1 & $4,00 \%$ \\
\hline & BE LIKELY TO & 2 & D0,002 \% & 0 & $\mathrm{X}$ \\
\hline & BE LIABLE TO & 1 & D0,001 \% & 0 & $\mathrm{x}$ \\
\hline & BE PRONE TO & 1 & D0,001 \% & 0 & $\mathrm{X}$ \\
\hline & BE SUPPOSED TO & 1 & D0,001 \% & 0 & $\mathrm{X}$ \\
\hline & BE DUE TO & 3 & d0,003\% & 0 & $\mathrm{X}$ \\
\hline TOTAL & & 693 & $0,7 \%$ & 465 & $1,7 \%$ \\
\hline
\end{tabular}

Annexe 2 : Adverbes d'intensité compris dans les citations des communiqués de presse de Greenpeace

\begin{tabular}{|l|l|l|l|l|}
\hline $\begin{array}{l}\text { Adverbes d'intensité } \\
\text { apparaissant dans le } \\
\text { corpus }\end{array}$ & $\begin{array}{l}\text { Nombre des adverbes } \\
\text { d'intensité dans le } \\
\text { corpus }\end{array}$ & $\begin{array}{l}\text { Nombre d'adverbes } \\
\text { d'intensité dans les } \\
\text { citations }\end{array}$ & $\begin{array}{l}\text { Ratio } \\
\text { citations/ } \\
\text { corpus }{ }^{\mathbf{4}}\end{array}$ \\
\hline absolutely & 12 & 9 & $75,0 \%$ \\
\hline all but & 1 & 1 & $100,0 \%$ \\
\hline badly & 3 & 1 & $33,3 \%$ \\
\hline barely & 1 & 1 & $100,0 \%$ \\
\hline certainly & 2 & 1 & $50,0 \%$ \\
\hline
\end{tabular}




\begin{tabular}{|c|c|c|c|}
\hline clearly & 25 & 15 & $60,0 \%$ \\
\hline comprehensively & 1 & 0 & $0,0 \%$ \\
\hline critically & 6 & 0 & $0,0 \%$ \\
\hline deeply & 5 & 3 & $60,0 \%$ \\
\hline definitely & 1 & 1 & $100,0 \%$ \\
\hline desperately & 2 & 2 & $100,0 \%$ \\
\hline dramatically & 4 & 1 & $25,0 \%$ \\
\hline drastically & 1 & 0 & $0,0 \%$ \\
\hline enough & 19 & 12 & $63,2 \%$ \\
\hline entirely & 14 & 10 & $71,4 \%$ \\
\hline especially & 11 & 4 & $36,4 \%$ \\
\hline essentially & 1 & 0 & $0,0 \%$ \\
\hline exactly & 5 & 1 & $20,0 \%$ \\
\hline exceedingly & 1 & 0 & $0,0 \%$ \\
\hline exceptionally & 2 & 0 & $0,0 \%$ \\
\hline exhaustively & 1 & 0 & $0,0 \%$ \\
\hline extremely & 8 & 1 & $12,5 \%$ \\
\hline fiercely & 1 & 0 & $0,0 \%$ \\
\hline firmly & 1 & 1 & $100,0 \%$ \\
\hline fully & 20 & 8 & $40,0 \%$ \\
\hline fundamentally & 2 & 2 & $100,0 \%$ \\
\hline greatly & 3 & 2 & $66,7 \%$ \\
\hline hardly & 1 & 1 & $100,0 \%$ \\
\hline heavily & 12 & 3 & $25,0 \%$ \\
\hline highly & 13 & 4 & $30,8 \%$ \\
\hline hugely & 1 & 1 & $100,0 \%$ \\
\hline incredibly & 3 & 3 & $100,0 \%$ \\
\hline
\end{tabular}




\begin{tabular}{|c|c|c|c|}
\hline irreparably & 1 & 0 & $0,0 \%$ \\
\hline just & 58 & 22 & $37,9 \%$ \\
\hline largely & 2 & 0 & $0,0 \%$ \\
\hline mainly & 10 & 1 & $10,0 \%$ \\
\hline mostly & 3 & 2 & $66,7 \%$ \\
\hline notably & 2 & 1 & $50,0 \%$ \\
\hline notoriously & 1 & 1 & $100,0 \%$ \\
\hline obviously & 3 & 1 & $33,3 \%$ \\
\hline only & 107 & 47 & $43,9 \%$ \\
\hline particularly & 1 & 0 & $0,0 \%$ \\
\hline perfectly & 1 & 1 & $100,0 \%$ \\
\hline pretty & 1 & 1 & $100,0 \%$ \\
\hline prominently & 1 & 0 & $0,0 \%$ \\
\hline quite & 1 & 1 & $100,0 \%$ \\
\hline really & 8 & 7 & $87,5 \%$ \\
\hline severely & 2 & 1 & $50,0 \%$ \\
\hline sharply & 1 & 0 & $0,0 \%$ \\
\hline significantly & 10 & 2 & $20,0 \%$ \\
\hline simply & 18 & 11 & $61,1 \%$ \\
\hline so & 10 & 7 & $70,0 \%$ \\
\hline specifically & 1 & 0 & $0,0 \%$ \\
\hline strongly & 9 & 5 & $55,6 \%$ \\
\hline substantially & 2 & 1 & $50,0 \%$ \\
\hline terribly & 1 & 1 & $100,0 \%$ \\
\hline thoroughly & 2 & 0 & $0,0 \%$ \\
\hline too & 19 & 12 & $63,2 \%$ \\
\hline totally & 4 & 3 & $75,0 \%$ \\
\hline
\end{tabular}




\begin{tabular}{|l|l|l|l|}
\hline truly & 10 & 2 & $20,0 \%$ \\
\hline utterly & 4 & 3 & $75,0 \%$ \\
\hline very & 29 & 19 & $65,5 \%$ \\
\hline virtually & 3 & 0 & $0,0 \%$ \\
\hline widely & 2 & 0 & $0,0 \%$ \\
\hline wildly & 2 & 1 & $50,0 \%$ \\
\hline woefully & 1 & 0 & $0,0 \%$ \\
\hline TOTAUX & $\mathbf{4 9 9}$ & $\mathbf{2 4 1}$ & $\mathbf{4 8 , 3} \%$ \\
\hline
\end{tabular}

\section{NOTES}

1. Le Trésor de la Langue Française informatisé (TLFi) est mis à la disposition des internautes par I'UMR ATILF.

2. Les segments en italiques au sein des citations (hormis les termes d'origine étrangère à l'anglais ou au français) ont été soulignés par nos soins dans l'ensemble de l'article.

3. Selon le Dictionnaire d'analyse du discours (Charaudeau \& Maingueneau 2002: 42), cette discipline cherche à étudier « le langage comme activité ancrée dans un contexte produisant des unités transphrastiques »: en d'autres termes, l'analyse du discours porte sur l'étude du discours, soit l'« usage de la langue dans un contexte particulier» (ibid. : 186).

4. Selon le glossaire du Centre de recherche et d'information socio-politiques (<http:// www.vocabulairepolitique.be/>), Greenpeace est une ONG internationale dite militante: elle organise des actions directes pour défendre une cause, à l'inverse des ONG dites humanitaires qui viennent directement en aide à des populations opprimées.

5. Par « citation », nous entendons l'action de retranscrire dans un premier discours un discours extérieur, attribué à un énonciateur distinct de l'énonciateur du premier discours, de façon totale ou partielle.

6. <http://www.greenpeace.org/international/en/>

7. <http://www.greenpeace.org/international/en/about/>

8. Le 18 septembre 2013, les vingt-huit militants écologistes et les deux journalistes qui composaient l'équipage de l'Arctic Sunrise, l'un des bateaux de Greenpeace International, ont été arrêtés lors d'une expédition en Arctique par l'armée russe qui les accusait d'avoir enfreint les règles internationales de navigation. Ils ont été emprisonnés en Russie jusqu'en décembre 2013.

9. <http://www.bbc.com/news/world-europe-24238251>

$<$ http://www.aljazeera.com/news/europe/2013/09/russia-grills-greenpeace-

activists-20139259918455863.html>

<http://www.reuters.com/article/2013/09/25/us-russia-greenpeace-idUSBRE98009I20130925>

10. Les semi-modaux sont dotés de fonctions similaires ou proches de celles des auxiliaires modaux. G. Leech (2004: 68) classe notamment parmi les semi-modaux be going to, be to, had better, have got to, have to, need to, be supposed to, used to et want to, en précisant toutefois que cette liste n'est pas limitative.

11. Peut-être à cause de l'impératif de concision auquel sont soumis les auteurs des communiqués de presse (les négations en no étant plus concises que les négations en not...any). 
12. "Quoting someone or using another's words allow a distant or 'distal' positioning of the reporter with respect to the content and sources of information ».

13. Les vingt-huit militants et les deux journalistes retenus en Russie furent tout d'abord accusés de piraterie qui, selon le communiqué 6, est punie de quinze ans de prison (« They now face a charge of piracy - an absurd charge that carries a maximum 15 year jail sentence »); puis de hooliganisme, crime pour lequel ils encouraient sept ans de prison selon le communiqué 1 («Russian authorities today formally charged a Greenpeace International activist with hooliganism, a crime that carries a maximum seven year jail term »).

14. Les pourcentages donnés ici ont été arrondis à la décimale la plus proche.

\section{RÉSUMÉS}

Cette étude cherche à identifier la visée communicationnelle et les spécificités éventuelles des communiqués de presse de l'ONG Greenpeace International à travers l'analyse macrostructurelle et microstructurelle des passages au discours direct, qui occupent une part essentielle des communiqués. L'étude porte sur 200 communiqués de presse publiés sur le site international de Greenpeace, et plus particulièrement sur les 564 citations au discours direct qu'ils comprennent. L'analyse montre que la plupart de ces citations, tout en étant attribuées à des sources extérieures, émanent en réalité des auteurs des communiqués. Ces "pseudo-citations" constituent également un trait caractéristique des communiqués de presse d'entreprise. Elles y sont destinées à encourager la reprise des communiqués par les journalistes en leur donnant une apparence neutre et en accentuant la ressemblance entre les communiqués et les articles de presse. L'autre visée communicationnelle principale de ces citations constitue l'un des traits distinctifs de notre corpus : le discours direct, qui véhicule les propos des militants et joue sur le pathos, est également censé rallier le lecteur aux causes défendues par l'organisation et l'inciter à agir.

This article aims to identify the communicative purpose and the potential specificity of the press releases published by Greenpeace International, an activist NGO, through a macro- and microanalysis of passages containing direct speech, which is frequently used in press releases. The study focuses on 200 press releases published on Greenpeace's international website, and more specifically on 564 passages resorting to direct speech. The analysis shows that most of the quotes were created by the authors of the press releases, although they are attributed to external sources. Greenpeace thus appears to make use of "pseudo-quotes" as corporate press releases do. They are used to give a neutral appearance to the press release and to strengthen its similarity to press articles, in order to encourage journalists to draw on the press release when writing their own reports. Our study identified a second communicative purpose that distinguishes the press releases in our corpus from corporate ones: direct speech quotations, which convey the activists' statements and sometimes rely on pathos, are also intended to entice the readers to fight for the causes supported by the organization. 
INDEX

Mots-clés : communiqué de presse, crédibilité, ONG militante, discours direct, persuasion, préformulation, stratégie rhétorique

Keywords : activist NGO, credibility, direct speech, press release, persuasion, preformulation, rhetorical strategy

\section{AUTEUR}

\section{MELWENN CLOAREC}

Melwenn Cloarec est agrégée d'anglais et diplômée de l'École normale supérieure de Cachan. Elle enseigne en classes préparatoires. melwenncloarec@hotmail.fr 